ratio of $1.50 \pm 0.08$ should be obtained at sea level for cosmic ray muons of energy $>10^{11} \mathrm{eV}$ assuming the accepted primary mass composition. This charge ratio value is significantly higher than the actual measured value at sea level of 1.30. This actual value indicates therefore that $\sim 25 \%$ of the total primary nucleon flux must be made up of neutrons. (The primary nucleon flux includes all nucleons whether in multi-nucleon nuclei or not). In other words the flux of heavy nuclei in primary cosmic rays at energies $\gtrsim 10^{12} \mathrm{eV}$ per nucleon must be larger than that observed at lower energies.

So this conclusion supports the earlier suggestion from the satellite work of Grigorov et al. Such a conclusion if confirmed will be of the utmost importance to cosmic ray physicists.

\section{Ribsomal switches}

from E. G. Richards

THE notion that RNA molecules can exist in several metastable conformational states and that such conformers can be separated by electrophoresis on polyacrylamide gels is by now well known. It all began with tRNA; 5S RNA was the next to exhibit this property and more recently, 16S RNA has been observed to do the same. So far the transformation from one conformer to another has required heat or reagents such as magnesium ions or EDTA. The idea that proteins could do this has remained speculative though proteins have been described that destabilise double helical regions in RNA structures.

These speculations have recently been turned into demonstrations in a paper by Hochkeppel and Craven (J. molec. Biol. 113, 623; 1977). It had previously been shown that $16 \mathrm{~S}$ RNA isolated from the $30 \mathrm{~S}$ ribosomal subunits of Escherichia coli by treatment with phenol (16S RNA ${ }^{p}$ ) was not the same as the RNA isolated by treatment with acetic acid and urea (16S RNA ${ }^{\mathrm{a}}$ ): the two forms can be separated by electrophoresis in agarose/polyacrylamide and $16 \mathrm{~S} \mathrm{RNA}^{\mathrm{a}}$ bound ribosomal proteins that $16 \mathrm{~S} \mathrm{RNA}^{\mathrm{p}}$ did not. In their latest paper they show that if they bind several of the ribosomal proteins to $16 \mathrm{~S} \mathrm{RNA}^{p}$, and then remove them again, there results RNA migrating with a mobility close to that of the slower 16S RNA* rather than that of the faster moving 16S RNA ${ }^{p}$. Appropri-

E. G. Richards is in the Department of Biophysics, King's College, London. ate controls established that it was not the re-extraction procedure itself that wrought this change. Proteins found to have this effect were S4, S7 and S8, but this of course does not preclude that other untried ones do the same.

These authors went on to demonstrate in a similar way that $16 \mathrm{~S} \mathrm{RNA}^{\mathrm{p}}$ pretreated with $\mathrm{S} 7$ (the protein being bound to the RNA and then removed) was able to bind $\mathrm{S} 9$ whereas untreated 16S RNA ${ }^{p}$ was not. 16S RNA $^{p}$ pretreated with both $\mathrm{S} 4$ and $\mathrm{S} 7$ was even more efficient at binding $\mathrm{S} 9$ but pretreatment with $\mathrm{S} 4$ alone did not affect the capacity to bind S9 even though it resulted in a lowered mobility. It would therefore seem that the different ribosomal proteins are able to bring about several different conformational states, with differential protein binding ability but all with reduced mobility.

Further experiments demonstrated that the RNA extracted from the reconstitution intermediate (RI) migrated slowly like $16 \mathrm{~S} \mathrm{RNA}^{\mathrm{a}}$ while that extracted from the heat activated particle ( $\left.\mathrm{RI}^{*}\right)$ migrated with the faster mobility. It was similarly shown that inactivation by ion depletion of intact $30 \mathrm{~S}$ ribosomal subunits results in the extraction of RNA with a lowered mobility as well as a loss of capacity to bind tRNA in the intact particle.

Present understanding suggests that the difference between one RNA conformer and another must reside either in the pattern of base-paired helical regions constituting the secondary structure or in the pattern of folding and interaction deployed in the tertiary structure. It thus seems likely that to change one conformer into another, base pairs or tertiary interactions must first be undone. Several proteins are able to bring this about (including the ribosomal protein $\mathrm{S} 1$ ) so it is not too surprising that the ribosomal proteins, S4, S7 and S8 can do likewise. Nevertheless, the implications for the study of ribosomes are very interesting indeed.

As Hochkeppel and Craven themselves point out, it is now possible to begin to give a rational interpretation in terms of conformational changes in the RNA of the assembly maps presented by Mizushima and Nomura. One can envisage a series of conformational switches each turned on by the binding of a specific protein and each enabling the next protein to bind. If this speculation turns out to be valid, the assembly of a ribosome must be seen as a carefully orchestrated process rather than a series of random associations occurring in no fixed sequence.

The possible importance of conformational switches was discussed a few months ago by Weidner et al. (Nature $266,193 ; 1977)$ in a discussion of the possible conformational states of 5S RNA, another ribosomal component. Recently Bear et al. (Nucleic Acids Res. 4, 2511; 1977) have presented some evidence that the binding of proteins L18 and L25 to 5S RNA wreaks a change in the RNA conformation. The evidence is based on changes on the circular dichroism spectra and melting curves of the RNA and RNA-protein complexes and is not wholely conclusive. It does, however, nudge one's prejudices a little further along the direction of supposing that conformational switches may be an important and widely used trick employed in a variety of dynamic molecular processes in living cells.

\section{New sulphide mineral discovered}

\section{from Peter J. Smith}

A PREVIOUSLY unknown potassiumrich sulphide mineral has been found by Clarke et at. (Earth planet Sci. Lett., 35, 421; 1977) within a clinopyroxene-ilmenite intergrowth from kimberlite diatremes in South Africa. Until the new sulphide has been more thoroughly investigated, Clarke and his colleagues in Canada and Scotland refrain from giving it a name, referring to it for the time being simply as FSKS (Frank Smith K-sulphide) after the Cape Province diamond mine of which the diatremes form the base. The discovery of a new mineral is not, of course, a completely unheard of event even at this late stage in the development of geology; but what makes FSKS of more than passing interest is that it may indicate an impontant source of potassium in the upper mantle and throw some light on the vexed question of the transfer of potassium and sulphur from the mantle to the core.

FSKS occurs as ovoid blebs (which also contain pyrrhotite and pentlandite), both as individual grains in cracks in the host clinopyroxene and, more commonly, in close association with the ilmenite lamellae. Because of its high potassium content (8.12-13.01 weight $\%$ ) it inevitably invites comparison with other potassium-bearing sulphides, namely, the djerfisherite found in several meteorites, the more copper-rich djerfisherite found in the Talnakh copper deposit in Russia, and the rasvumite found in the Russian Khobina massif. But chemical analysis shows FSKS to be quite different from

Peter J. Smith is a reader in the Department of Earth Sciences at the Open University. 